\title{
Variable Universe Fuzzy Controlled Incremental Conductance MPPT for Z-source PV Inverter
}

\author{
Jingwei Zhang ${ }^{1}$, Honghua Wang ${ }^{1, *}$, Chengliang Wang ${ }^{2}$ and Rong Sun ${ }^{3}$ \\ ${ }^{1}$ College of Energy and Electrical Engineering, Hohai University, Nanjing 211100, Jiangsu Province, China \\ ${ }^{2}$ Jiangsu Frontier Electric Technology Co., Ltd., Nanjing 211100, Jiangsu Province, China \\ ${ }^{3}$ Jiangsu Electric Power Company Research Institute, Nanjing 211100, Jiangsu Province, China \\ * Corresponding author
}

\begin{abstract}
Maximum power point tracking (MPPT) is always a hot research area for photovoltaic (PV) system. In this paper, based on high efficiency $Z$-source $P V$ inverter, a variable universe fuzzy controlled incremental conductance (Inc-Cond) is proposed for MPPT. After designed fuzzy sets and rules, parameters of exponential-type and proportional-type stretching functions are investigated in detail. The influence to system performance are also analyzed. Corresponding simulations of Z-source PV system are implemented in MATLAB/Simulink. Simulation results show that the selection of parameter values has significant impact on system response speed and steady precision. Finally, an example is given to verify the feasibility of proposed MPPT algorithm. Compared with conventional fuzzy controlled Inc-Cond, voltage and power of PV array are more stable during MPPT. Inverter output voltage is stable and its THD is approximate $0.86 \%$.
\end{abstract}

Keywords-variable universe fuzzy controller; photovoltaic; incremental conductance; $Z$-source inverter

\section{INTRODUCTION}

With the development of sustainable energy industry, photovoltaic (PV) system has become a popular research area in recent decades. A high efficiency energy harvesting system is widely desired by system designers, especially a high efficiency maximum power point tracking (MPPT) method for PV system. Conventional methods include: fractional opencircuit voltage method, perturb and observe (P\&O), incremental conductance (Inc-Cond), et al. [1]. Some improved MPPT method based on intelligent algorithm were also applied, including artificial neural network and fuzzy PID controllers [2,3]. Variable universe fuzzy PID controller is a type of adaptive fuzzy control which has dynamic adjustable universe. Thus, it has higher steady precision and is also applied for PV boost converters [4].

Z-source inverter has proposed in recent years, which has less power devices compared with conventional two-stage inverter, which makes it have higher efficiency and compact structure [5,6]. In this paper, the principle of Z-source inverter is introduced. Different from normal fuzzy PID control, a variable universe fuzzy controller is designed to produce adaptive variable voltage step size for incremental conductance. After comparing the performance of different stretching functions, they are applied respectively for MPPT of Z-source PV inverter. Simulation results show that parameters of stretching functions has significant influences on system response speed and steady precision.

\section{PRINCIPLE OF Z-SOURCE INVERTER}

As Figure 1 shows, Z-source network is formed by inductors and capacities connected in X-type, which utilizes shoot-through duty of inverter bridge to boost output voltage. The output voltage of capacitors in Z-source and its output voltage $U_{Z}$ are $[5,6]$ :

$$
\left\{\begin{array}{l}
U_{C}=\frac{1-D_{0}}{1-2 D_{0}} U_{P V} \\
U_{Z}=\frac{1}{1-2 D_{0}} U_{P V}=B U_{P V}
\end{array}\right.
$$

$U_{P V}$ denotes output voltage of PV array, $U_{C}$ represents the voltage of capacitance in Z-source network, $U_{Z}$ is output voltage of Z-source network, $D_{0}$ depicts the shoot-through duty, $B$ is the boost factor of Z-source network. Equation (1) indicates $U_{P V}$ can be boosted via adjusting shoot-through duty in the range of $[0,0.5)$, and generated power of PV array can be regulated via controlling voltage of PV array to track the maximum power point. In this paper, the SVPWM is improved for inserting shoot-through time during inverter modulation.

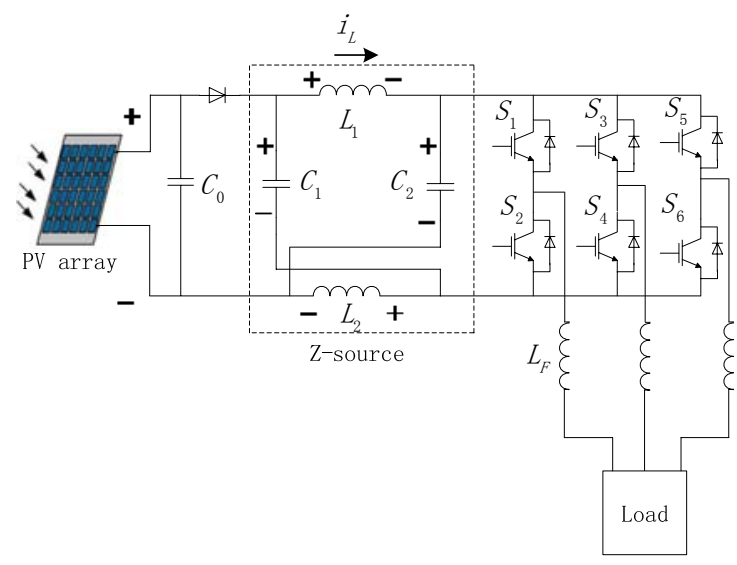

FIGURE I. STRUCTURE OF Z-SOURCE PV INVERTER. 
III. Design of VARIABle Universe FuZzy CONTROLLED INCREMENTAL CONDUCTANCE

\section{A. Design of Fuzzy Controller}

Conventional incremental conductance (Inc-Cond) method utilizes the derivative of PV panel power $P_{P V}$ and voltage to judge the direction of MPPT [1], as equation (2) shows:

$$
\frac{\mathrm{d} P_{P V}}{\mathrm{~d} U_{P V}}=\frac{\mathrm{d}\left(I_{P V} U_{P V}\right)}{\mathrm{d} U_{P V}}=I_{P V}+U_{P V} \frac{\Delta I_{P V}}{\Delta U_{P V}}
$$

The derivative is zero at maximum power point (MPP). If the derivative is positive, voltage $U_{P V}$ should be increased. If the derivative is negative, $U_{P V}$ should be reduced. Hence it determines the direction of increased voltage step.

However, the voltage step size of conventional Inc-Cond is constant. A greater step size can accelerate response speed but has larger oscillation around MPP, so the variable voltage step size is necessary for Inc-Cond to achieve faster response and higher steady precision. Hence variable universe fuzzy controller is designed to produce variable step size, as Figure 2 shows. The inputs of fuzzy controller are the latest voltage step size $V_{\text {step last }}$ and the deviation of power $d P$. Controller output is the step size in current sampling period, $V_{\text {step }}$. They are both divided into 6 fuzzy sets, including: negative big (NB), negative medium (NM), negative small (NS), positive small (PS), positive medium (PM) and positive big (PB). In this paper, two 50W PV panels are used to form the PV array, open-circuit voltage of PV array is approximate $43 \mathrm{~V}$, so the range of step size $V_{\text {step last }}$ and $V_{\text {step }}$ is defined as [-12,12], the range of $d P$ is $[-20,20]$. Triangular functions are applied as membership functions, as shown in Figure 3.

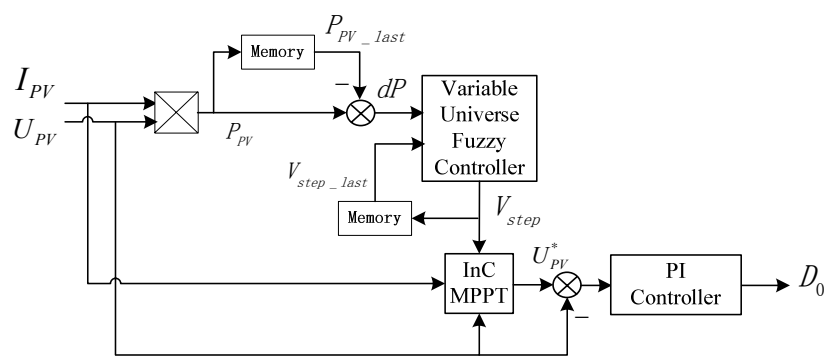

FIGURE II. CONTROL DIAGRAM OF PROPOSED VARIABLE UNIVERSE FUZZY CONTROLLED INCREMENTAL CONDUCTANCE

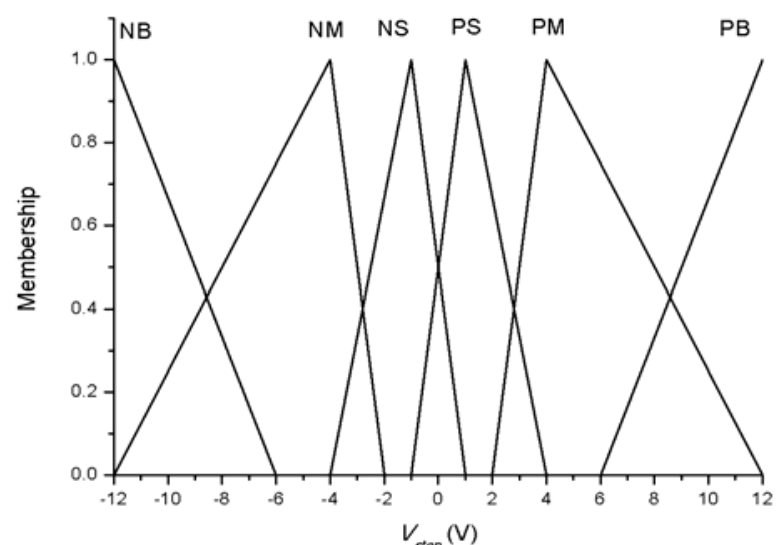

(A)

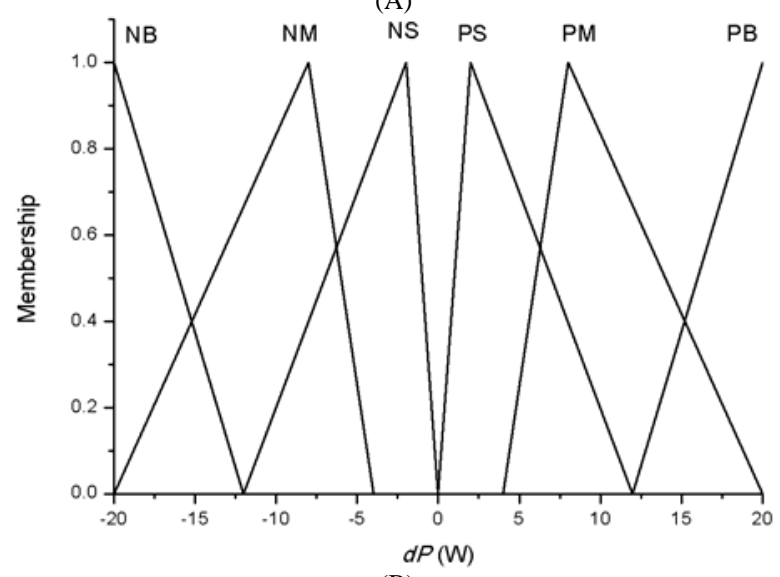

(B)

FIGURE III. MEMBERSHIP FUNCTION OF INPUTS AND OUTPUT, (A)

$$
V_{\text {STEP LAST, }} V_{\text {STEP, }} \text { (B) DP }
$$

Then fuzzy rules are designed considering the output characteristic of PV panel, as shown in Table I. Different voltage step size should be chosen at different sides of MPP due to the power-voltage $(\mathrm{P}-\mathrm{V})$ curve of $\mathrm{PV}$ array is asymmetric. So greater voltage step size should be used when operating point locates at left side of MPP, and less step size should be applied when operating point is at right side of MPP So the fuzzy rules table is not strictly symmetrical. Additionally, a commonly used centroid method is used for defuzzification.

TABLE I. DESIGNED FUZZY RULES TABLE

\begin{tabular}{|c|c|c|c|c|c|c|}
\hline \multirow{2}{*}{$\boldsymbol{d P}$} & \multicolumn{6}{|c|}{$\boldsymbol{V}_{\text {step_last }}$} \\
\cline { 2 - 7 } & NB & NM & NS & PS & PM & PB \\
\hline NB & PB & PM & PS & NS & NM & NM \\
\hline NM & PM & PS & PS & NS & NS & NM \\
\hline NS & PM & PS & PS & NS & NS & NM \\
\hline PS & NM & NS & NS & PS & PS & PM \\
\hline PM & NM & NS & NS & PS & PS & PM \\
\hline PB & NM & NS & NS & PS & PM & PB \\
\hline
\end{tabular}

B. Analysis of Variable Universe Fuzzy Control

In this paper, the variable universe fuzzy control is applied for MPPT of Z-source PV inverter. Commonly, stretching 
functions are utilized to shift the universe of membership functions. The exponential-type and proportional-type contraction-expansion factor are usually used in stretching functions. Assuming the universe of controller input is $[-E, E]$, $e$ represents the input variable, the contraction-expansion factor $\alpha(e)$ changes universe to $[-\alpha(e) E, \alpha(e) E]$. The exponential-type factor is:

$$
\alpha_{\text {exp }}(e)=1-\lambda \exp \left(-k e^{2}\right), \lambda \in(0,1), k>0
$$

$\lambda$ and $k$ are parameters of stretching function, which influence fuzzy controller performance significantly. The proportionaltype factor is:

$$
\alpha_{\text {prop }}(e)=\left(\frac{|e|}{E}\right)^{\tau}+\varepsilon, \tau>0, \varepsilon>0
$$

$\tau$ and $\varepsilon$ are also corresponding parameters. These two types of contraction-expansion factor have respective advantages. Firstly, the exponential-type factor is analyzed, the influence of two parameters $\lambda$ and $k$ are investigated independently. As shown in Figure 4(a), keeping parameter $k$ as 0.1, the value of $\lambda$ is set to $0.1,0.5,0.9$ respectively and stretching functions are plotted. Curves of stretching functions indicate that a greater $\lambda$ leads the factor achieve less value, which makes the value of factor reduce faster and more sensitive when system is under steady state. Besides, in Figure 4(b), keeping the value of $\lambda$ as 0.9 , a smaller value of $k$ makes the curve much smooth, and leads the compressed universe change continually in the range of input. Hence a larger $\lambda$ and smaller $k$ are recommended.

Then, the influence of parameters to proportional-type factor are investigated. In Figure 5(a), keeping $\varepsilon$ constant, $\tau$ influences the shape of function curve significantly, which is similar to parameter $k$ for exponential-type factor. And $\varepsilon$ leads the whole curve move upward in the axis, as Figure 5(b) shows. Hence a smaller value of $\varepsilon$ is recommended.

Comparing with proportional-type factor, the exponentialtype factor achieves a sharper stretching function curve and makes the universe of membership much sensitive to inputs, which produce higher steady precision and less oscillation. However, in the application of fuzzy controlled Inc-Cond MPPT, adjustment of stretching function parameters are more complicated. Thus, some simulations are necessary.

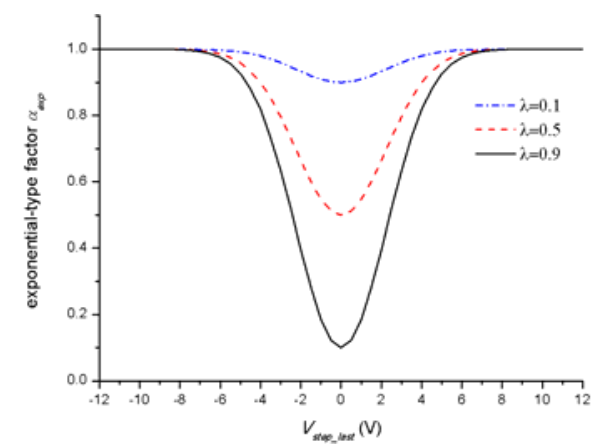

(A)

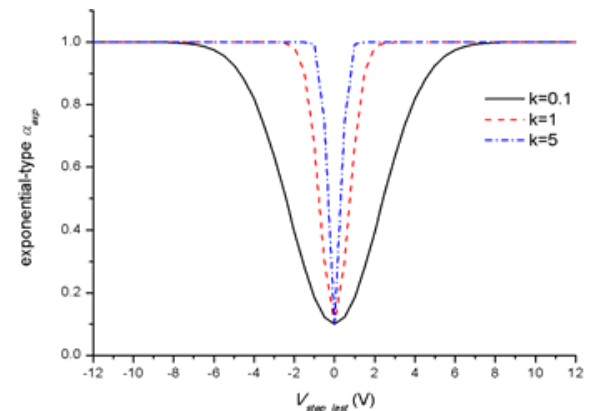

(B)

FIGURE IV. INFLUENCE OF PARAMETERS TO EXPONENTIAL-TYPE FACTOR, (A) PARAMETER $\Lambda$, (B) PARAMETER $K$

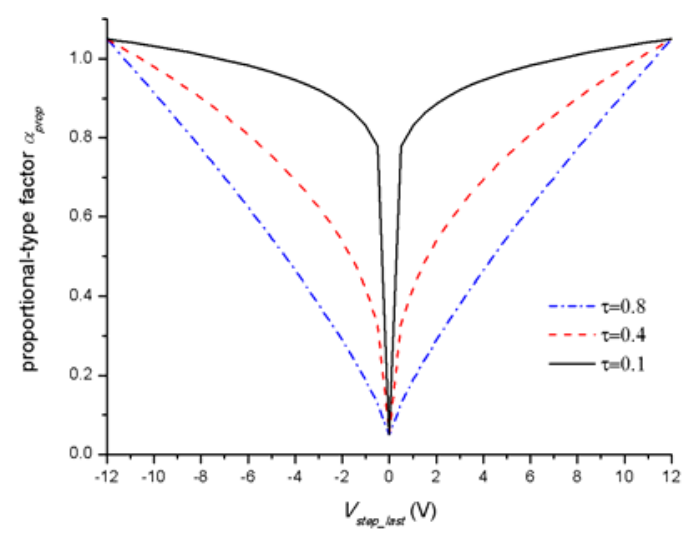

(A)

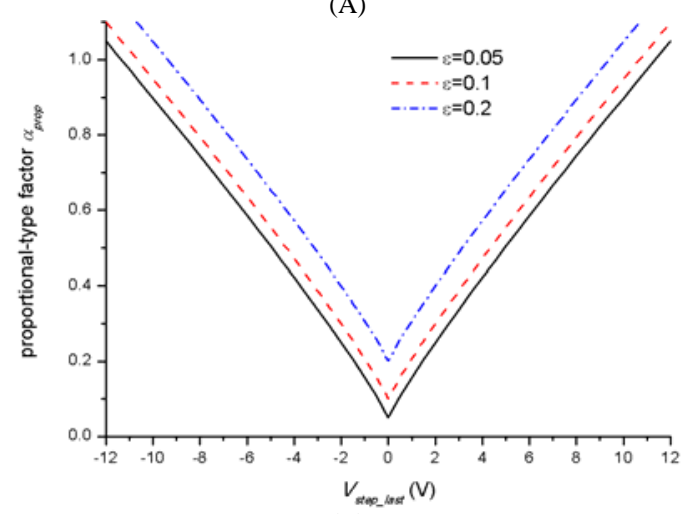

(B)

FIGURE V. INFLUENCE OF PARAMETERS TO PROPORTIONALTYPE FACTOR, (A) PARAMETER $T$, (B) PARAMETER $E$

\section{SimUlation AND RESUlts}

Focusing on the influence of parameters to system performance, some simulations were implemented in MATLAB/Simulink. As mentioned above, the PV array is formed by two 50W PV panels connected in series. Electrical characteristics of used PV panel are listed in Table II. Considering voltage and current ripple of capacitors and inductors, parameters of Z-source network are designed as: $L_{1}=L_{2}=1 \mathrm{mH}, \quad C_{1}=C_{2}=330 \mathrm{uF}$. Irradiance is $1 \mathrm{~kW} / \mathrm{m}^{2}$ and temperature is $25^{\circ} \mathrm{C}$ during simulation. 
TABLE II. ELECTRICAL CHARACTERISTICS OF PV MODULE

\begin{tabular}{|c|c|}
\hline Parameters & Value \\
\hline Power & $50 \mathrm{~W}$ \\
\hline Open circuit voltage, $\mathrm{V}_{\mathrm{OC}}$ & $21.5 \mathrm{~V}$ \\
\hline Short circuit current, $\mathrm{I}_{\mathrm{SC}}$ & $2.95 \mathrm{~A}$ \\
\hline Voltage at MPP, $\mathrm{V}_{\mathrm{m}}$ & $18.4 \mathrm{~V}$ \\
\hline Current at MPP, $\mathrm{I}_{\mathrm{m}}$ & $2.72 \mathrm{~A}$ \\
\hline Test irradiance & $1000 \mathrm{~W} / \mathrm{m}^{2}$ \\
\hline Test temperature & $25^{\circ} \mathrm{C}$ \\
\hline
\end{tabular}

Figure 6 gives the simulation results of MPPT controlled voltage of PV array, $U_{P V}$, and the stretching function of membership universe uses exponential-type factor. Different values of parameter $k$ are applied. As Figure 6(a) shows, the smaller $k$ is, the faster the voltage step size converge to zero. A smaller step size leads voltage changes slowly during MPPT. Nevertheless, a greater parameter $k$ may lead voltage step size insensitive to controller inputs, which makes step size too large and causes voltage oscillation. Hence, $k=1$ is more appropriate than other two values.

Besides, as Figure 6(b) reveals, with the increment of parameter $\lambda$, variable universe fuzzy controlled Inc-Cond achieves higher steady precision, and voltage of PV array is much more stable, which agrees with analysis mentioned before. Hence, in this system, a greater $\lambda$ is necessary, but the parameter $k$ should be adjusted to make the voltage converge stably during MPPT.

The proportional-type factor is also applied in stretching function for Z-source PV inverter, and its performance is analyzed. Figure 7(a) shows that the greater value of $\varepsilon$ makes system response faster but produce vibration in steady state. Hence, a medium value is appropriate. In addition, in Figure 7(b), simulation results show that a greater value of $\tau$ leads universe compress much faster and generates less voltage step size. Hence it costs more time for PV array voltage converge to MPP. However, if parameter $\tau$ is too small, the voltage step size would keep a greater value and make the voltage vibrate around the MPP. So a proper value of $\tau$ should be chosen for proposed variable universe fuzzy controlled Inc-Cond MPPT. For instance, setting $\tau$ to 0.7 and $\varepsilon$ is 0.1 , the voltage and output power of PV array are shown in Figure 8, DC power is stable at $100 \mathrm{~W}$, i.e. the maximum power. A comparison with conventional fuzzy controlled Inc-Cond is also implemented. The voltage step size converge at $1.35 \mathrm{~V}$ for conventional fuzzy controlled Inc-Cond, inevitably causes voltage and power vibration. Inverter output voltage in A phase is shown in Figure 9, THD of A phase voltage is $0.86 \%$, which verifies the feasibility of proposed variable universe fuzzy controlled MPPT and application in Z-source PV inverter.

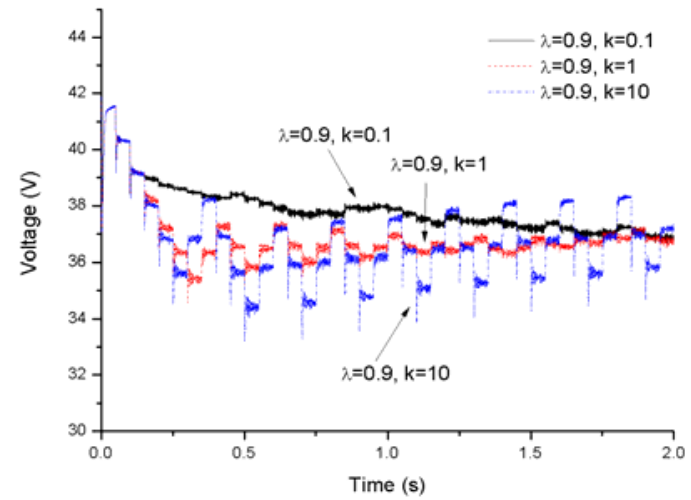

(A)

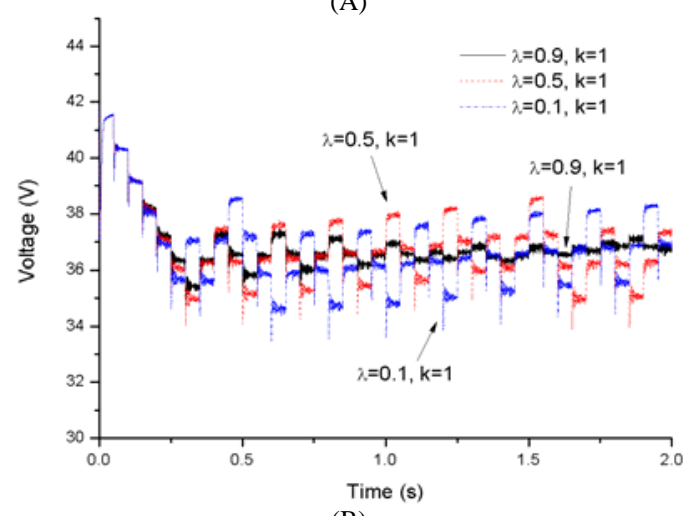

(B)

FIGURE VI. VOLTAGE CURVES OF PV ARRAY UNDER DIFFERENT PARAMETERS, (A) PARAMETER $K$, (B) PARAMETER $A$

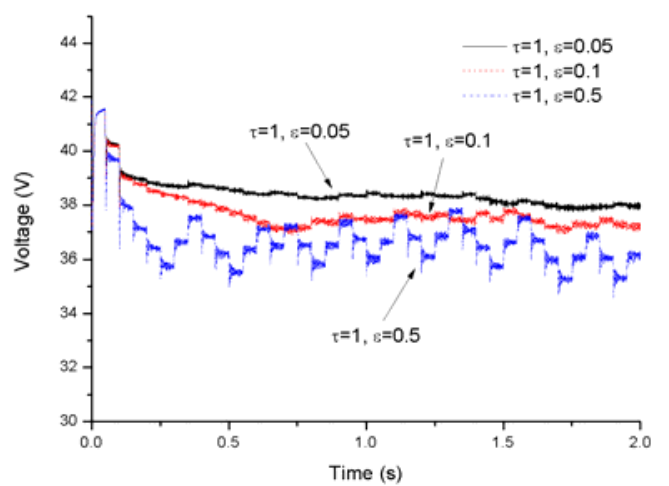

(A)

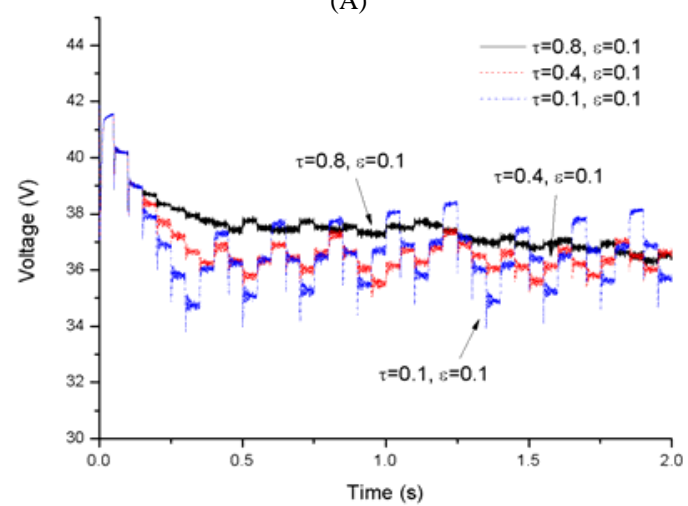

(B)

FIGURE VII. VOLTAGE CURVES OF PV ARRAY UNDER DIFFERENT PARAMETERS, (A) PARAMETER $\varepsilon$, (B) PARAMETER $\tau$ 

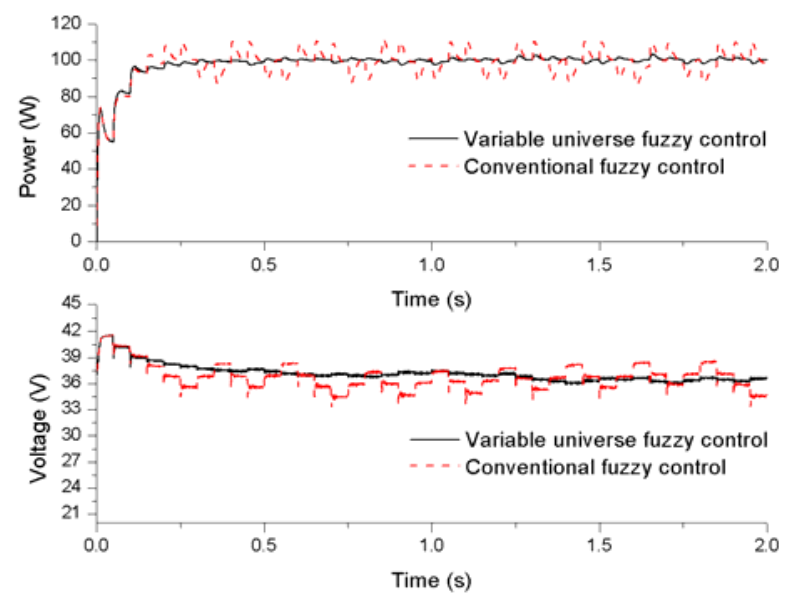

FIGURE VIII. OUTPUT POWER AND VOLTAGE OF PV ARRAY
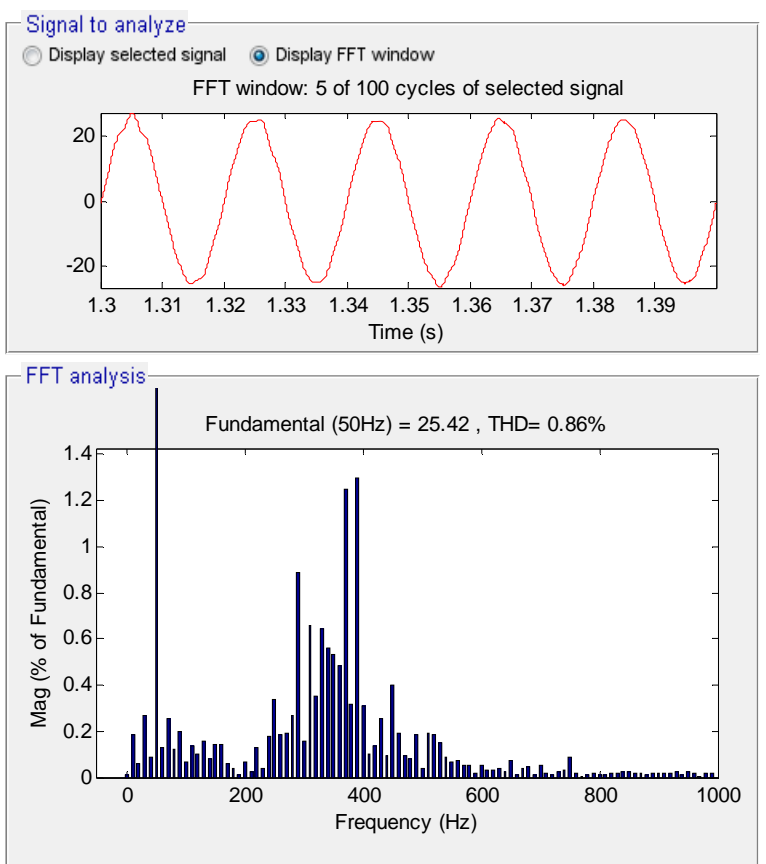

FIGURE IX. INVERTER OUTPUT VOLTAGE IN A PHASE AND FFT RESULT

\section{CONCLUSION}

In this paper, a variable universe fuzzy controlled MPPT for Z-source inverter is proposed. Corresponding membership functions and fuzzy rules of controller are designed. After investigation of stretching functions, including exponentialtype and proportional-type factors, parameter influences to stretching functions are illustrated. Analysis results indicate that a larger $\lambda$ and smaller $k$ are recommended to achieve higher steady precision for exponential-type factor, and a medium $\tau$ and smaller $\varepsilon$ is necessary for proportional-type factor. Corresponding simulation results reveal that fuzzy controller output should not converge too fast because controller output $V_{\text {step }}$ influences searching speed and steady precision of Inc-Cond during MPPT. Different parameters are applied independently and analyzed. Finally, an example and comparison is given to verify feasibility of proposed variable universe fuzzy controlled Inc-Cond MPPT for Z-source PV inverter, corresponding inverted A phase voltage is stable and its THD is $0.86 \%$.

\section{ACKNOWLEDGMENT}

This research was supported by a grant from the State Grid Jiangsu electric power company project of China (No.J2014028).

\section{REFERENCES}

[1] B. Subudhi, R. Pradhan, "A comparative study on maximum power point tracking techniques for photovoltaic power system," IEEE Trans. Sustainable Energy, vol. 4, no. 1, pp. 89-98, Jan. 2013.

[2] M. Veerachary, T. Senjyu, K. Uezato, "Neural-network-based maximum-power-point tracking of coupled-inductor interleaved-boostconverter-supplied PV system using fuzzy controller," IEEE Trans. Industrial Electronics, vol. 50, no. 4, pp. 749-758, Aug. 2003.

[3] C. H. Hsieh, "Optimal fuzzy-immune-PID controllers design of PWM DC-DC converters,” presented at the NAFIPS’ 07. Annual Meeting of the North American. San Diego, CA, Jun. 24-27, 2007.

[4] Z. Yang, Y. Chen, J. Hu, "The research on application of variable universe fuzzy control to maximum power point tracking system,” presented at the 3rd International Conference on PESA 2009. Hong Kong, May 20-22, 2009.

[5] F. Z. Peng, A. Joseph, J. Wang, et al., "Z-source inverter for motor drives,” IEEE Trans. Power Electronics, vol. 20, no. 4, pp. 857-863, Jul. 2005.

[6] K. Q. Qu, Q. Q. Niu, C. Yang, J. B. Zhao, "Battery charge-discharge control strategy based on the single Z-source three-level SVPWM inverter," presented at the 2013 IEEE Int. Conf. on Applied Superconductivity and Electromagnetic Devices (ASEMD), Beijing, China, Oct. 25-27, 2013. 